\title{
Channel Characteristics of Rail Traffic Tunnel Scenarios Based on Ray-Tracing Simulator
}

\author{
Jinmeng Zhao $\mathbb{D}^{1},{ }^{1}$ Lei Xiong $\left(\mathbb{D},{ }^{1}\right.$ Danping He, ${ }^{1}$ and Jiadong $\mathrm{Du}^{2}$ \\ ${ }^{1}$ State Key Laboratory of Rail Traffic Control and Safety, Beijing Jiaotong University, China \\ ${ }^{2}$ The China Academy of Information and Communications Technology (CAICT), China \\ Correspondence should be addressed to Lei Xiong; lxiong@bjtu.edu.cn
}

Received 30 March 2018; Accepted 27 June 2018; Published 1 August 2018

Academic Editor: Sana Salous

Copyright (c) 2018 Jinmeng Zhao et al. This is an open access article distributed under the Creative Commons Attribution License, which permits unrestricted use, distribution, and reproduction in any medium, provided the original work is properly cited.

\begin{abstract}
The tunnel scenario is a major rail communication scenario. In this paper, the radio channel characteristics of tunnel scenarios with different carrier frequencies, different distances between the transmitter $(\mathrm{Tx})$ and receiver $(\mathrm{Rx})$, and cross sections are simulated with a ray-tracing tool. Key parameters such as path loss, Rician K-factor, root mean square (RMS) delay spread, and angular spread are studied. According to the results, higher frequencies introduce larger path loss and the presence of the vehicle body increases the path loss by about $35 \mathrm{~dB}$ in the scenario; at the same time it will also cause the fluctuation and instability of the path loss. Besides, the influence of reflections from the side walls is significant on radio propagation. The channel experiences more severe fading in a narrow tunnel compared with others.
\end{abstract}

\section{Introduction}

The rail traffic communications have experienced a rapid development recently, such as high-speed railway, municipal railway, and urban railway system. The rail traffic scenario is indispensable for both private and public mobile communications. It is widely agreed that the wireless channel model is significant to carry out mobile communications research, system development, and network deployment, and so on [1]. Currently, the Long-Term Evolution Railway (LTER) system has been recommended to replace the global system for mobile communication railway (GSM-R) system for high-speed train (HST) communication system as a part of intelligent transportation systems (ITS) [2,3]. In addition, the research on 5th-Generation wireless systems (5G) based on high-speed railway (HSR) has become a trend to meet the need of transmission capabilities [4-6]. Thus the study on channel models in rail traffic system for new communication system is indispensable.

The wireless channel research needs to be carried out for various typical rail traffic scenarios (viaducts, tunnels, cuttings, etc.), considering the significant differences between the rail traffic scenarios and the public network scenarios [7].
Field test and ray tracer (RT) are two well-known methods for implementing channel characterization. Field test in the field of rail traffic is hard, costly, and long-term. RT provides a means of accurately prediction of the wave propagation which is timesaving and convenient. Therefore, RT is widely used in channel modeling for confined environments.

Tunnel scenario is one of the most common scenarios, especially in the mountainous and hilly areas. Considering the unique construction, the wireless propagation in tunnel scenarios is different from other HST scenarios which attracts a lot of research interests [8-10]. Leaky cables and Distributed Antenna System (DAS) [11, 12] are mainly two promising methods to provide radio coverage in tunnels. Several tunnel channel models were proposed in recent years, such as raytracing model, multimode model, and propagation-graph theory based model $[13,14]$. A real multipath propagation model for radio transmission in typical rectangular subway tunnel was presented in [15] using RT to analyze Doppler spread. The three-dimensional (3D) models of six scenario modules for mmWave and $\mathrm{THz}$ train-to-infrastructure channels were defined and constructed for the first time in [16], which considered reality obstacle objects. Based on the wideband measurements conducted in the tunnel scenario 
by using the mobile hotspot network system, the authors in [17] explored key channel characteristics in different HSR scenarios by 3D RT. Besides, suggestions on symbol rate, subframe bandwidth, and polarization configuration were provided to guide the $5 \mathrm{G}$ mmWave communication system design in typical HSR scenarios.

The aforementioned research summarized that the majority of existing works contribute to modeling methods based on large-scale parameters. However, previous models were analyzed and compared based on field test. The measurements results such as channel impulse response (CIR) are mixed with a variety of factors. In addition, measurements focusing on narrowband single-input single-output (SISO) systems at low frequency band result in missing multipath parameters such as angular spread and Doppler shift. There is still an urgent demand for an accurate complete channel model considering large-scale parameters, small-scale parameters, and spatial parameters $[18,19]$ for multitype tunnel scenarios in SISO systems or multiple-input multipleoutput (MIMO) systems. Based on RT, channel parameters such as the path loss, multipath delay, and angular spread can be obtained exactly and analyzed. This paper provides the simulation results in different type of tunnels at $1.8 \mathrm{GHz}$. Optimized deployment recommendations are provided for MIMO systems and antenna configuration.

The rest of the paper is organized as follows. The existing channel models for the tunnel scenario of 3GPP are introduced in Section 2. The defined tunnel scenarios and simulation setup are presented in Section 3. Key parameters such as path loss, Rician K-factor, root mean square (RMS) delay spread, and angular spread in tunnels with different cross sections at different frequencies are analyzed in Section 4. Conclusions are drawn in Section 5.

\section{Channel Models}

3GPP TSG-RAN WG4 in RAN\#66 meeting defined four typical high-speed railway scenarios. A variety of standard channel models in tunnel scenarios were adopted by $3 \mathrm{GPP}$ [20-23], like the single tap channel, time delay line (TDL) model, etc.

Scenarios $2 \mathrm{c}$ and $2 \mathrm{~g}$ are corresponding to tunnel scenarios covered by leaky cables. The coverage of the Evolved Node $\mathrm{B}(\mathrm{eNB})$ is extended by deploying leaky cables in the $2 \mathrm{~g}$ and 2 c scenarios, which is shown in Table 1 . In scenario $2 \mathrm{~g}$, the User Equipment (UE) communicates directly with the leaky cable in the tunnel. The signal is severely attenuated due to the shielding of the train body in this case. In scenarios $2 c$, relays are set up on the train. The UE achieves two-hop communication with the leaky cable through relay as shown in Figure 1. Since the transmit power gradually attenuates along the leaky cable, RF amplifiers will typically be applied to ensure adequate signal strength. The propagation channel between the in-car relay and the UE does not involve highspeed movement.

3GPP R4-154106 proposes a one-tap channel model for leaky cables deployment in the tunnel scenario as shown in Figure 2, where $D_{0}$ is the distance between two neighbor

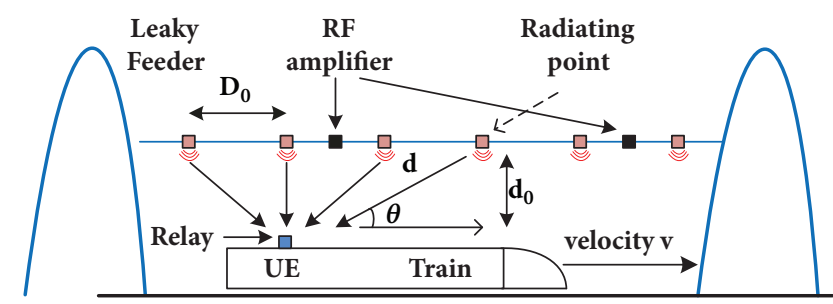

FIgURE 1: Leaky cable coverage with relay.

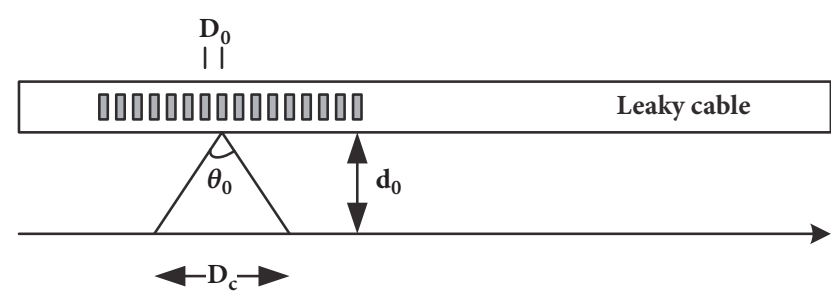

FIGURE 2: Detail illustration of leaky cable.

radiating points; the radiation angle of radiating point is denoted as $\theta_{0}$. It can be assumed that the receiver $(\mathrm{Rx})$ is covered by $8-9$ radiation spots. The RF receive signal is

$$
\begin{aligned}
r(t) & =\sum_{k} x(t) \sqrt{P_{k}} e^{j 2 \pi\left(f_{c}-f_{d, k}\right) t}+z(t) \\
& =x(t) e^{j 2 \pi f_{c} t} \sum_{k} \sqrt{P_{k}} e^{-j 2 \pi f_{d, k} t}+z(t)
\end{aligned}
$$

where $P_{k}$ denotes the normalized received power of the $k_{t h}$ radiation spot, $f_{d, k}$ is the respective Doppler shift, $z(t)$ is the received noise, and $x(t)$ is the transmitted signal. The power of the fading gain can be modeled as a Rician distribution based on the central limit theorem.

However, this model is only a simplified model. It ignores the reflection component and the scattering component which should be considered in a limited space. Besides, the parameters, such as tunnel size, cross section, internal electromagnetic (EM) properties of tunnel walls, surface roughness, and antenna polarization mode, affect the channel characteristics. RT views radio waves reflected by surfaces of the tunnel. It solves the problem of large workload and poor applicability in field test and also compensates for the inability of the traditional model to provide specific wireless channel parameters, such as amplitude, delay, Doppler shift, and angular spread, which is of great significance for wireless communication systems. It is an effective modeling method to establish a $3 \mathrm{D}$ channel model of the rail communication scenarios and analyze the radio signal propagation under different coverage patterns and multiple fading characteristics.

\section{Scenario Definition and Simulation Setup}

3.1. Tunnel Scenarios Definition. Given the different geological conditions, the cross section of the tunnel varied in reality. Four typical shapes of cross sections: rectangular, arched, long arched (combined rectangular and semicircular), and 
TABLE 1: Tunnel scenarios by leaky cables.

\begin{tabular}{lcc}
\hline Scenarios & Description & Notation \\
\hline $2 \mathrm{c}$ & The tunnel is equipped with a leaky cable & Two hop \\
& that communicates with the train's roof & RP and with the UE through the RP \\
$2 \mathrm{~g}$ & Laying leaky cables in the tunnel to \\
achieve direct coverage of the UE & One hop
\end{tabular}

semicircular, are shown in Figure 3. The side length of rectangular is $6 \mathrm{~m}$ in tunnel $a$; the height of arched is $6 \mathrm{~m}$, and the center of circle is $2 \mathrm{~m}$ high above the ground in tunnel $b$; tunnel c contains a rectangular whose side length is $4 \mathrm{~m}$ and a semicircular whose radius is $2 \mathrm{~m}$; the radius of semicircular is $6 \mathrm{~m}$ in tunnel $d$. The number of tunnel surface in the simulation is $4,10,9$, and 13 , respectively. Figure 4 shows the details of the simplified tunnel model referring to the metro tunnel project boundary map provided by the China Railway Fourth Survey and Design Institute Group Co., Ltd. The train model is shown in Figure 5. The 3D tunnel models in this work are built by Google SketchUp. These models are considered in the following simulation analysis.

3.2. Parameters Setup. The simulation parameters should be carefully determined to ensure that the simulation results are accurate and effective and make simulation energy-friendly and timesaving. RT supports a variety of radio wave propagation mechanisms, such as direction, reflection, scattering, diffraction, and transmission. Reflection order means that rays experience up to several times specular reflection from transmitter (Tx) to $\mathrm{Rx}$. The appropriate reflection order is necessary not only to reflect the actual reflectance, but also to avoid a longer simulation time.

Based on the above ideas, the channel characteristics under different reflection orders are simulated and analyzed in tunnel scenario in Figure 4 with all radio wave propagation mechanisms. The key channel characteristics, such as Rician $\mathrm{K}$-factor, delay spread, angle spread of angle of arrival (AOA), and angle of departure (AOD), are compared, respectively, under different reflection orders range from $6^{\text {th }}-9^{\text {th }}$. When the reflection order is set to higher than $6^{\text {th }}$, there is rarely a change. In addition, we define

$$
P L_{\text {error }}(i, j)=\frac{\left|P L_{i, f}-P L_{j, f}\right|}{P L_{j, f}}
$$

where the $P L_{i, f}$ is the path loss when the frequency is $f$ and the reflection order is $i$. The $P L_{\text {error }}(i, j)$ shows the difference rate between $i^{t h}$ and $j^{t h}$ order in a series of frequencies. The CDF of $P L_{\text {error }}$ is shown in Figure 6. The means of $P L_{\text {error }}(8$, 9), $P L_{\text {error }}(7,8)$, and $P L_{\text {error }}(6,7)$ are $4.26 \times 10^{-12}, 1.9 \times$ $10^{-2}$, and 0.18 , respectively. The $P L_{\text {error }}(8,9)$ is the smallest, ranging from $6.41 \times 10^{-13}$ to $1.72 \times 10^{-11}$. The $P L_{\text {error }}(7,8)$ ranges from $4.97 \times 10^{-5}$ to 0.12 . The $P L_{\text {error }}(6,7)$ ranges from $1.73 \times 10^{-4}$ to 0.53 , which is larger than others. As a result, the path loss up to higher than $8^{\text {th }}$ order is hardly change. Thus the $8^{\text {th }}$ order is adopted in simulation due to a tradeoff
TABle 2: Parameters setup.

\begin{tabular}{lc}
\hline Parameter & Value \\
\hline Frequency & $1.8 \mathrm{GHz} / 5.8 \mathrm{GHz}$ \\
Transmitting power & $25 \mathrm{dBm}$ \\
Antenna(Tx, Rx) & $\begin{array}{c}\text { Omnidirectional antenna } \\
\text { (vertically polarized) }\end{array}$ \\
Tx location & $(0,0,3)$ \\
Rx location & $(\mathrm{x}, 0,5)$ \\
Reflection order & 8 \\
Bandwidth & $10 \mathrm{MHz}$ \\
Resolution & $1 \mathrm{MHz}$ \\
\hline
\end{tabular}

between computational complexity and precision as shown in Table 2. Considering the fact that the tunnel scenarios are long straight tunnels covered by concrete with no vents and pipes and other obstacles, diffraction is irrelevant here as well as transmission because the Tx is not fixed in the wall.

Detailed parameters setup is shown in Table 2. The frequency is set to $1.8 \mathrm{GHz}$ and $5.8 \mathrm{GHz}$ in order to compare channel characteristics at different frequencies. Signal propagates with $25 \mathrm{dBm}$ of transmitting power. Tx is fixed at one end of the tunnel with the height of $3 \mathrm{~m}$. Rx moves along the $\mathrm{x}$-axis with the height of $5 \mathrm{~m}$. Both of them use vertically polarized omnidirectional antennas. The channel bandwidth $B=10 \mathrm{MHz}$, and the system time domain resolution $\Delta t=1$ / $B$. In order to better observe the path with the maximum excess delay max, the number of simulated frequency points $N_{f}$ should be determined enough [24]:

$$
\tau_{\max } \leq N_{f} \times \Delta t
$$

in which, in other words, the frequency domain resolution $\Delta f$ $=B / N_{f}$ should be set sufficiently.

\section{Simulation Results and Analyses}

In this section, the simulation results of tunnel channel characteristics in different cross sections and different frequencies are presented and analyzed. Furthermore, suggestions on optimized deployment are provided.

4.1. Large-Scale Parameters. The reliable large-scale channel models are essential to network deployment and optimization. Typically, the path loss is expressed as

$$
P L(d)=A+10 n \lg (d)+X
$$




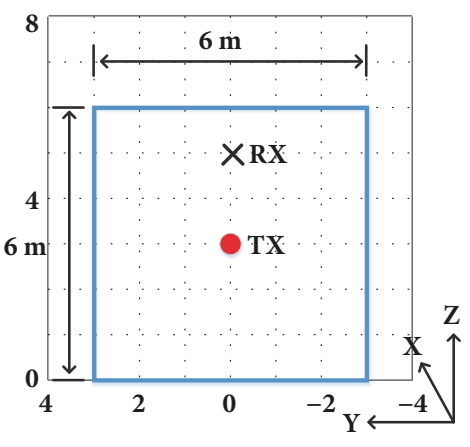

(a)

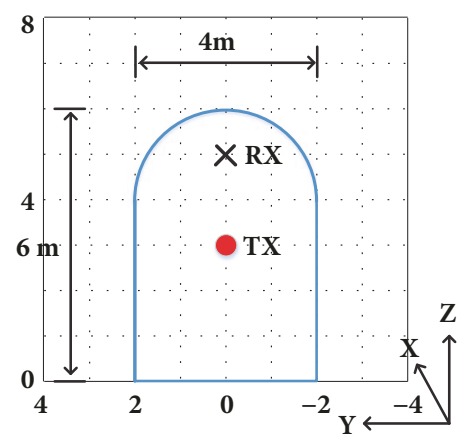

(c)

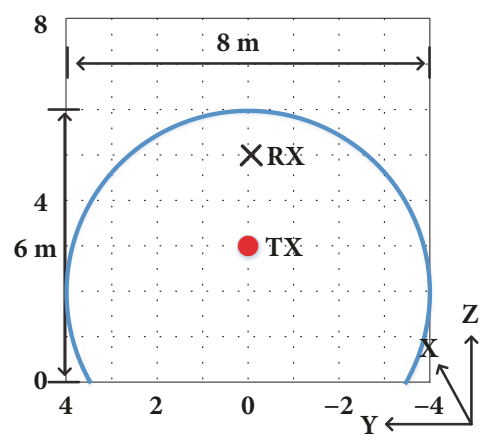

(b)

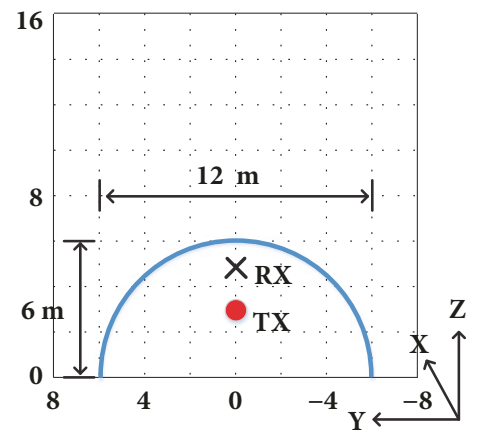

(d)

Figure 3: Regular cross sections for tunnels. (a) Rectangular. (b) Arched. (c) Long arched. (d) Semicircular.

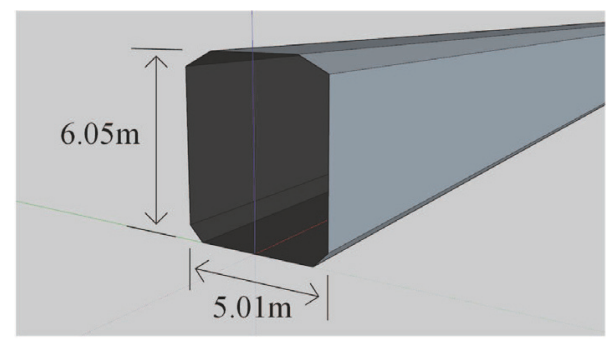

FIGURE 4: Simplified tunnel model (actual scenario).

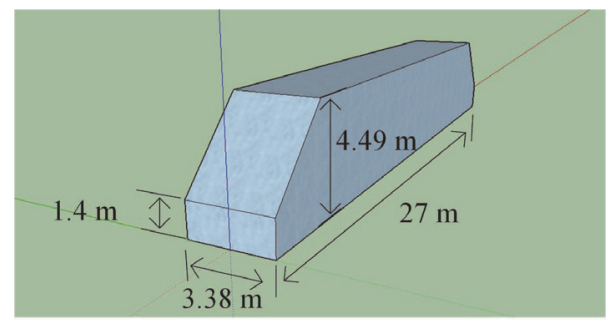

FIGURE 5: Train model for HST.

where the $P L(d)$ is path loss without small-scale fading and it is a function of the distance between Tx and $\mathrm{Rx}$ [25]. $n$ is path loss exponent, and $\mathrm{X}$ (shadow fading) is the zero-mean Gaussian random variable with standard deviation $\sigma$. Figure 7 shows the path loss at $1.8 \mathrm{GHz}$ in different cross sections. In the $0-190 \mathrm{~m}$ section, the path loss is nearly the same. However

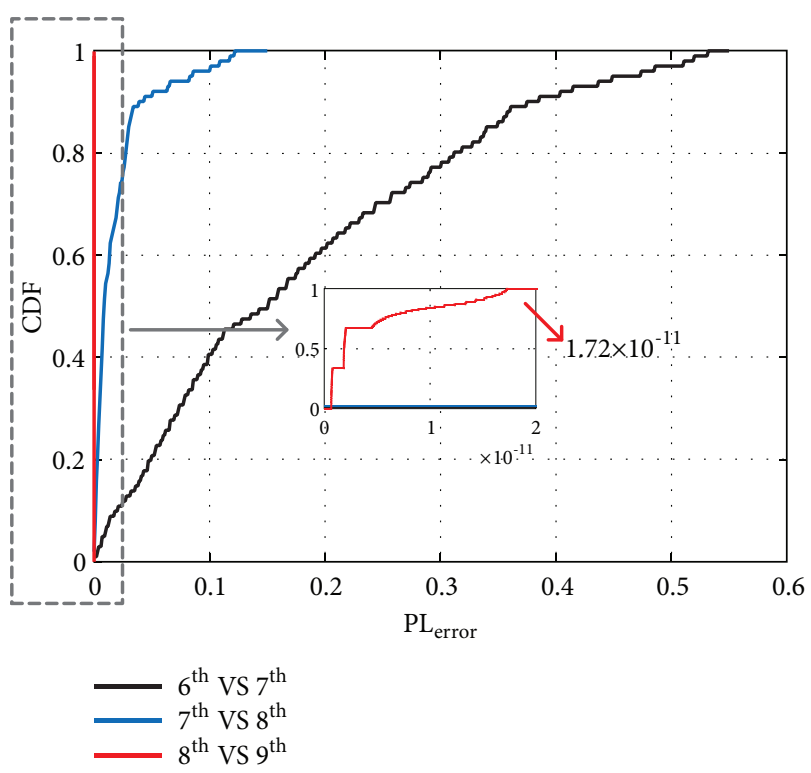

FiguRe 6: CDF of $P L_{\text {error }}$.

in the 190-500 $\mathrm{m}$ section, the path loss in tunnel $c$ has a higher path loss than other tunnels. The path loss in tunnel $c$ can be divided into two phases, and path loss exponents are 1.96 and 3.62, respectively, which means that there is a breakpoint $\left(d_{0}=190.10 \mathrm{~m}\right)$ in this scenario. These tunnel cross sections have the same height, and the width varies in shape and size. The path loss fitting result given in Table 3 


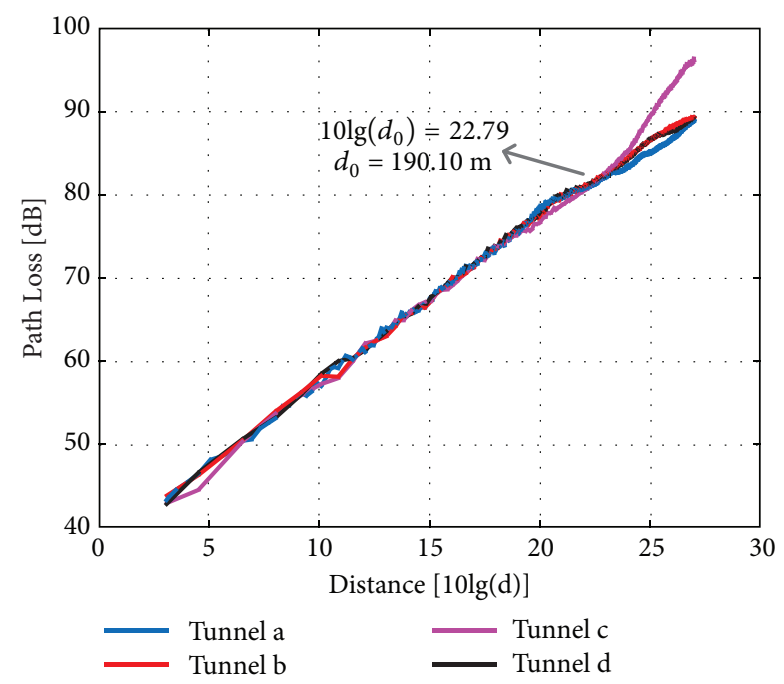

FIGURE 7: Path loss in different tunnel cross sections at $1.8 \mathrm{GHz}$.

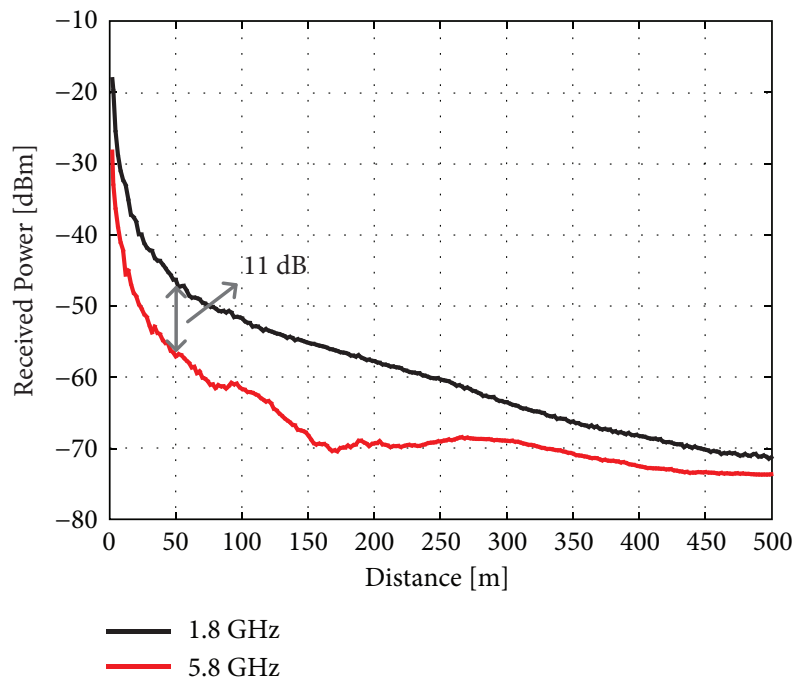

FIGURE 8: Received power at different frequencies in tunnel $c$.

indicates that the lateral shape has an important impact on the path loss in the empty tunnel with strong line of sight (LOS) path. The path loss exponent in tunnel $c$ is greater than 2 because of the existing breakpoint. The path loss exponents are $1.77,1.84$, and 1.88 in tunnel $a, d$, and $b$, respectively. Compared with the measurement results in straight arched tunnel $[26,27]$ and rectangular tunnel [28-30], the value of path loss exponent 1.96 in tunnel $c$ is similar to 1.40-2.03 at 954-2000 MHz in straight arched tunnel. Meanwhile, path loss exponent 1.77 in tunnel $a$ is similar to $1.65-1.94$ at $945-$ $2650 \mathrm{MHz}$ in rectangular tunnel. Simulation results in this paper are basically consistent with the measurement results in previous work.

Figure 8 shows the received power at different frequencies in tunnel $c$. As the frequency increases, the received power decreases. When the distance between Tx and Rx equals $50 \mathrm{~m}$, the received power at $1.8 \mathrm{GHz}$ is $11 \mathrm{~dB}$ higher than that at $5.8 \mathrm{GHz}$. Received power has larger fluctuation, and deeper

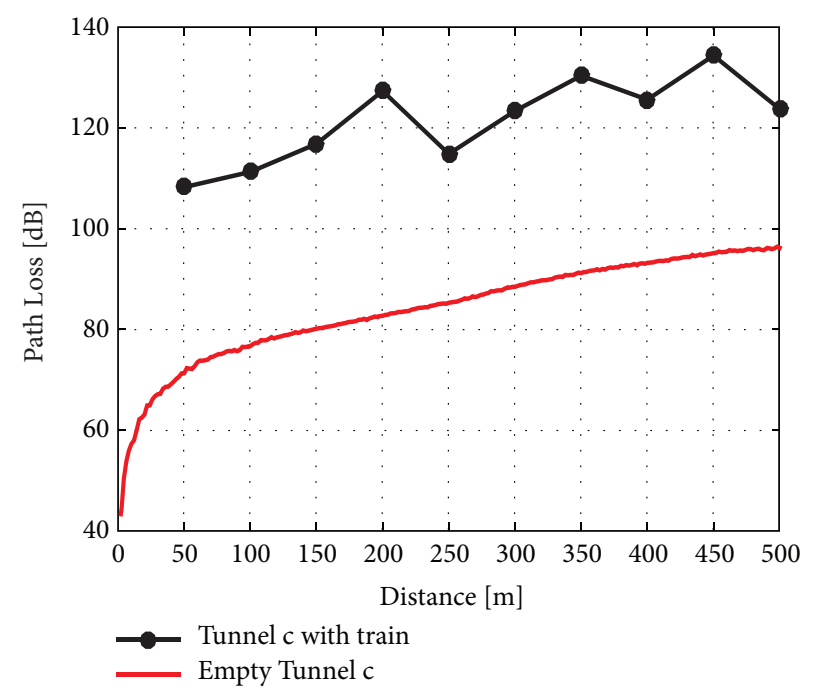

FIGURE 9: Path loss in empty tunnel and tunnel with train.

TABle 3: Path loss fitting result at $1.8 \mathrm{GHZ}$.

\begin{tabular}{lccc}
\hline Parameter & $\mathrm{A}$ & $\mathrm{n}$ & $\sigma$ \\
\hline Tunnel a & 41.29 & 1.77 & 0.74 \\
Tunnel b & 39.46 & 1.88 & 0.45 \\
Tunnel c & 31.82 & 2.30 & 1.60 \\
Tunnel d & 41.29 & 1.84 & 0.60 \\
\hline
\end{tabular}

TABle 4: Path loss fitting result in tunnel $c$.

\begin{tabular}{lccc}
\hline Parameter & $\mathrm{A}$ & $\mathrm{n}$ & $\sigma$ \\
\hline $1.8 \mathrm{GHz}$ & 31.82 & 2.31 & 1.60 \\
$5.8 \mathrm{GHz}$ & 51.51 & 1.78 & 1.45 \\
\hline
\end{tabular}

fading is prone to occur in higher frequency. Based on the similar simulation scenario and parameters setup, the same conclusion is also reflected on the simulation results at three different typical carrier frequencies, i.e., $900 \mathrm{MHz}, 2.45 \mathrm{GHz}$, and $5.75 \mathrm{GHz}$ in [31]. Table 4 shows the fitting parameters at different frequencies. The path loss exponent is 2.31 at 1.8 GHz, while the path loss exponent is less than 2 at higher frequency. Results confirm that the frequency band has a strong impact on the wireless propagation.

For the tunnel with train, the path loss result in selected locations is shown in Figure 9. Rx is fixed on the head of train when the train gradually moves away from the Tx. The obstruction and reflection of the train make the received signal experience longer distance and greater attenuation. The corresponding number of arriving rays reduces sharply. The path loss increases by $35.58 \mathrm{~dB}$ with the presence of the vehicle body. The result shows that the path loss in the empty tunnel scenario rises more steadily. Suggestions are given that the antenna deployment in reality scenarios should make sure of the existence of LOS path between Tx and Rx. Moreover, antenna placement is selected carefully to make the blocking effect of the train as small as possible for which further researches are needed. 


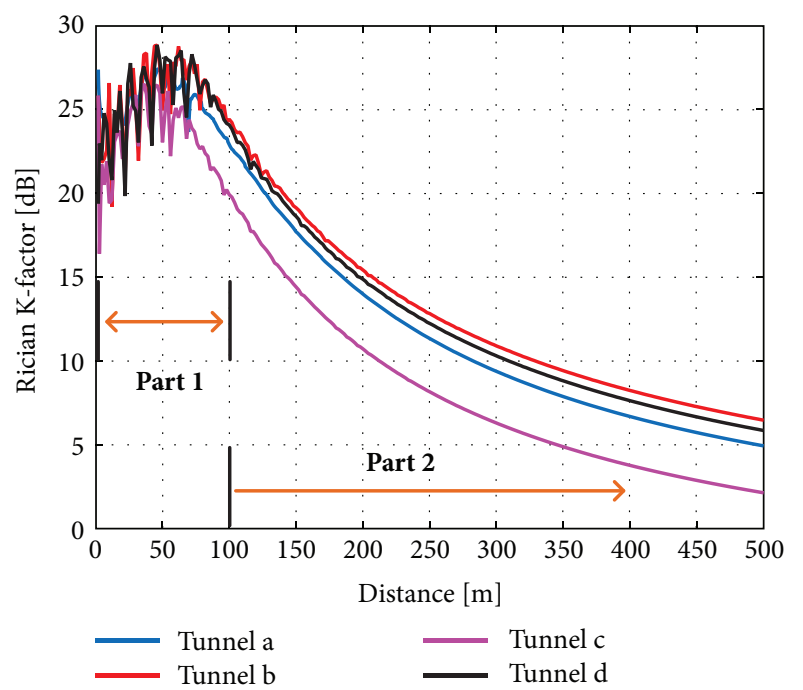

(a)

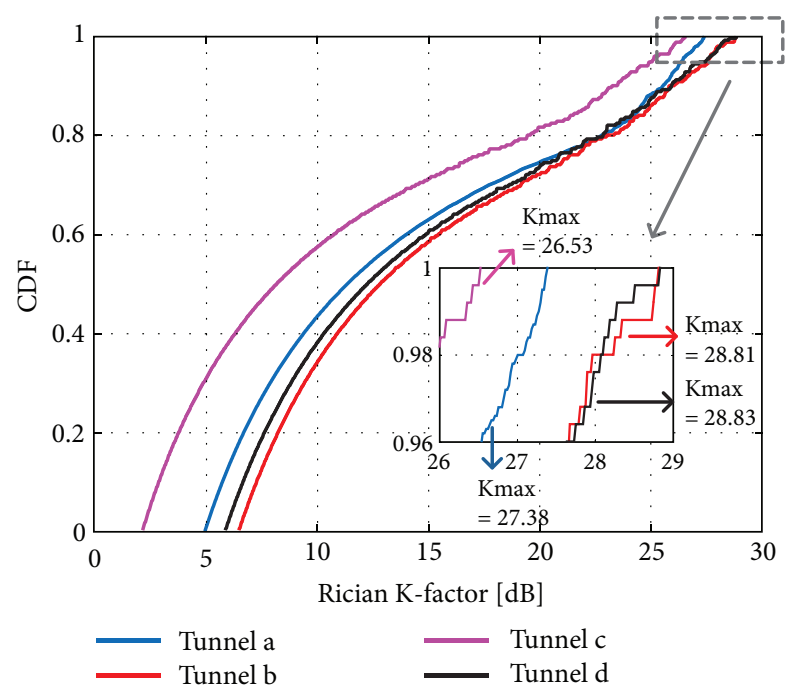

(b)

Figure 10: (a) Rician K-factor at 1.8 GHz. (b) CDF of Rician K-factor at all the snapshots.

TABLE 5: Small scale parameters.

\begin{tabular}{lcccc}
\hline Parameter & $\begin{array}{c}\text { Mean } \\
\text { K-factor } \\
{[\mathrm{dB}]}\end{array}$ & $\begin{array}{c}\text { Percentage } \\
\text { of K<25 dB }\end{array}$ & $\begin{array}{c}\text { Mean } \\
\text { delay spread } \\
\text { [ns] }\end{array}$ & $\begin{array}{c}95 \% \text { of } \\
\text { delay spread } \\
\text { range [ns] }\end{array}$ \\
\hline Tunnel a & 13.7 & $88 \%$ & 0.47 & $0.31-0.76$ \\
Tunnel b & 14.98 & $86 \%$ & 0.37 & $0.09-0.75$ \\
Tunnel c & 10.82 & $94.8 \%$ & 0.33 & $0.17-0.66$ \\
Tunnel d & 14.46 & $87 \%$ & 0.40 & $0.16-0.80$ \\
\hline
\end{tabular}

4.2. Small-Scale Parameters. Small-scale parameters have an important impact in wireless communication system design and analysis. Rician K-factor, RMS delay spread, and angular spread are key parameters to describe the small-scale characteristics. Table 5 shows the K-factor and RMS delay spread in different tunnel scenarios.

4.2.1. Rician K-Factor. Rician $\mathrm{K}$-factor is defined as the radio of the power of LOS path to the power of non-line-of-sight (NLOS) paths [32]. Figure 10 shows K-factor at $1.8 \mathrm{GHz}$ and CDF of K-factor at all the snapshots. Figure 10(a) depicts that the simulation section can be divided into two parts; K-factor fluctuates severely in part one $(0-100 \mathrm{~m})$ and then decreases smoothly in part two (100-500 m). In the first $50 \mathrm{~m}$, the fading is weak, but, after that, the fading is increasingly serious whatever the tunnel scenario is. Table 5 demonstrates that $94.8 \%$ of K-factor in all the snapshots in tunnel $c$ is lower than $25 \mathrm{~dB}$, but there is about $88 \%$ of $\mathrm{K}$-factor lower than $25 \mathrm{~dB}$ in other tunnel scenarios as shown in Figure 10(b). The mean Kfactor of tunnel scenarios is around $13.49 \mathrm{~dB}$ larger than the result $11.5 \mathrm{~dB}$ of empirical cluster characteristics extracted for tunnel scenarios at $2.14 \mathrm{GHz}$ [33]. Since the path loss increases with frequency, LOS path experiences a deeper fading at $2.14 \mathrm{GHz}$. In conclusion, channel characteristics in tunnel $c$ experience a severe fading than others.
4.2.2. RMS Delay Spread. Figure 11 shows that RMS delay spread at $1.8 \mathrm{GHz}$ and CDF of RMS delay spread at all the snapshots. Tunnel $a$ is the rectangular tunnel (W6 $\mathrm{m} \times \mathrm{H} 6 \mathrm{~m}$ ), and tunnel $c$ is the long arched tunnel (W4 $\mathrm{m} \times \mathrm{H} 6 \mathrm{~m}$ ). The most apparent difference between tunnel $a$ and tunnel $c$ is their widths. RMS delay spread in tunnel $a$ is larger than that in tunnel $c$ along the simulation section. In Table 5, we notice that RMS delay spread is lower than $0.8 \mathrm{~ns}$ in at least $95 \%$ at all the snapshots, and the maximum RMS delay spread is 1.18 ns. The results in Table 5 illustrate that $95 \%$ of the RMS delay spreads at all the snapshots in tunnel $a$ and tunnel $c$ are $0.45 \mathrm{~ns}$ and $0.49 \mathrm{~ns}$, respectively, which is narrow compared with other scenarios. On the other hand, mean RMS delay spread is the largest in tunnel $a$, and the weighted average of the delay spread in tunnel $c$ is the smallest. As a result, the channel in tunnel c experiences a more stable fading. The RMS delay spread varies in ns because of fewer obstacles in empty tunnel, which is similar to the measurement results of delay spread 2-27 ns in mine tunnels [34].

4.3. Spatial Parameters. The RMS angular spreads in tunnel $c$ and CDF of angular spreads are shown in Figure 12. ASA, ESA, ASD, and ESD are angular spreads of the azimuth angle of arrival, the elevation angle of arrival, the azimuth angle of departure, and elevation angle of departure, respectively. 


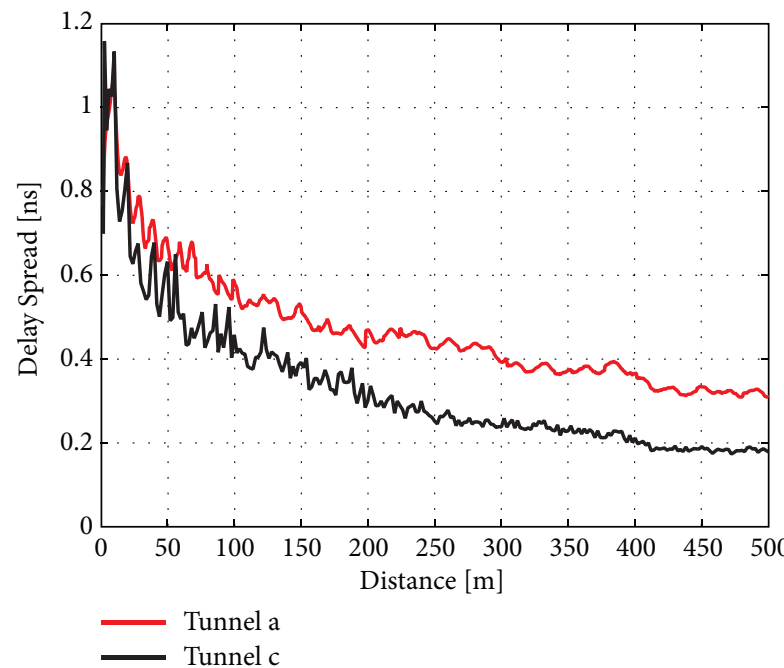

(a)

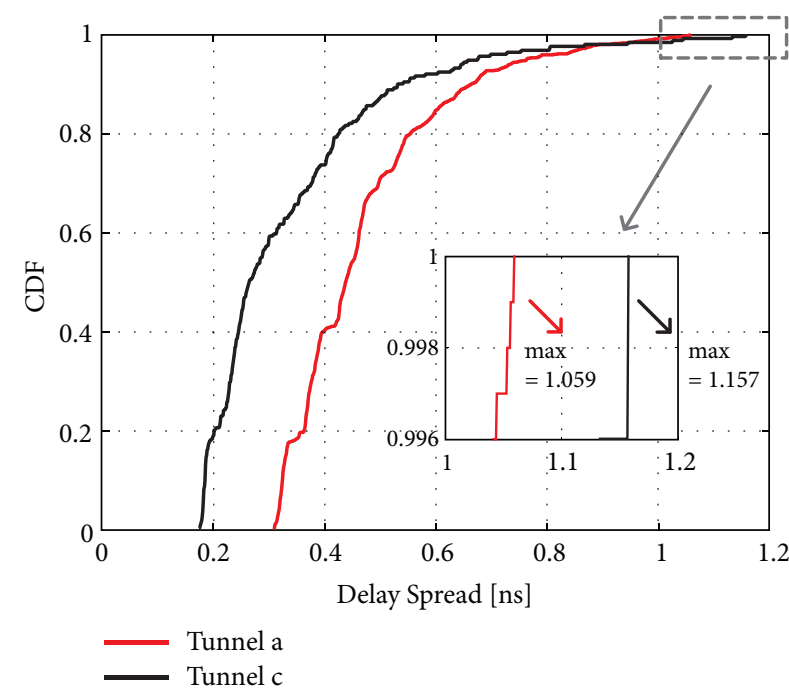

(b)

FIGURE 11: (a) RMS delay spread at $1.8 \mathrm{GHz}$. (b) CDF of RMS delay spread at all the snapshots.

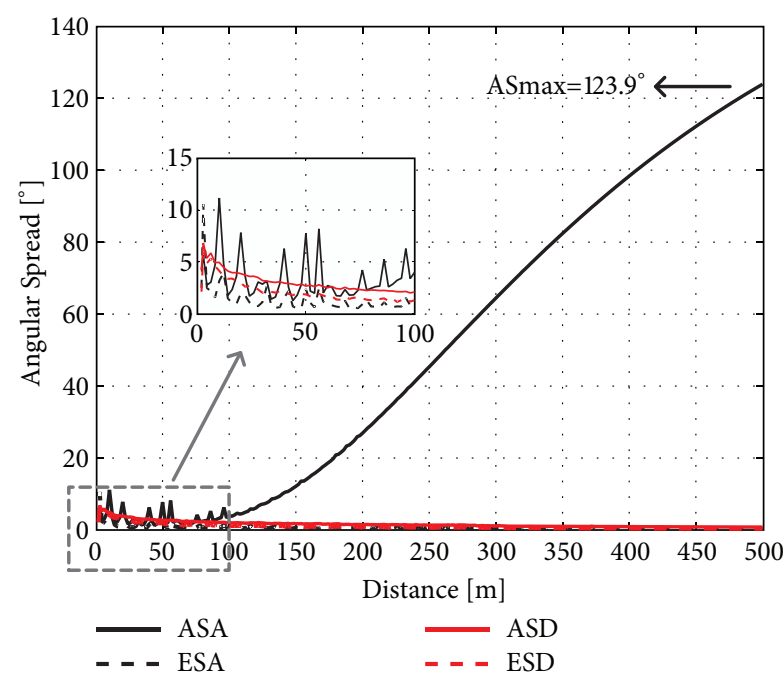

(a)

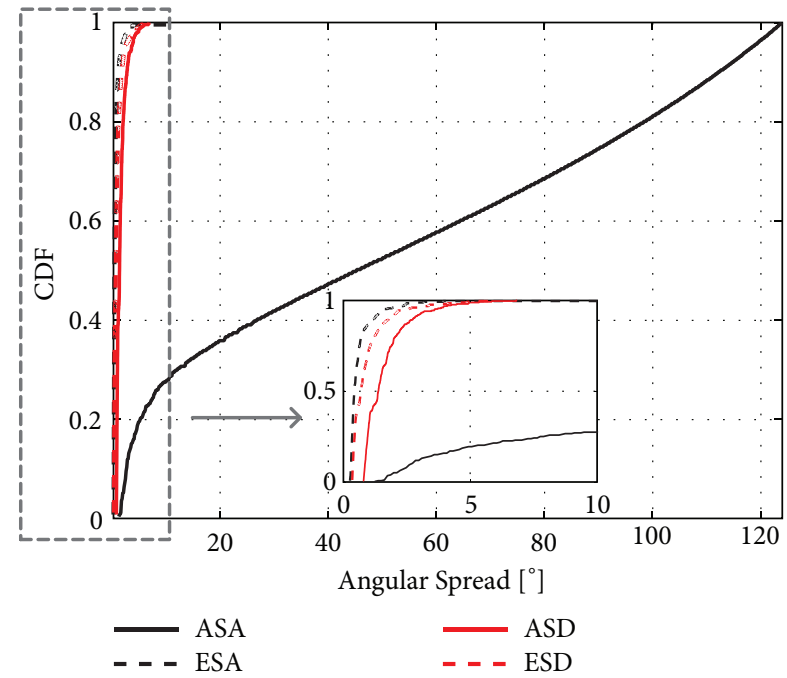

(b)

FIGURE 12: Tunnel c scenario. (a) RMS angular spread at $1.8 \mathrm{GHz}$. (b) CDF of RMS angular spread at all the snapshots.

Figure 11 illustrates that ESA, ASD, and ESD decrease slowly as the $\mathrm{Rx}$ moves far away from $\mathrm{Tx}$, and they are lower than $10.20^{\circ}$ at all the snapshots. The means of ASA, ESA, $\mathrm{ASD}$, and ESD are $51.25^{\circ}, 0.67^{\circ}, 1.63^{\circ}$, and $0.95^{\circ}$, respectively. $99 \%$ of ESA, ASD, and ESD are less than $5^{\circ}$. However, ASA varies differently compared with others. It gradually increases and the max ASA is $123.90^{\circ}$ when the distance between Tx and $\mathrm{Rx}$ reaches $500 \mathrm{~m}$. Angular spread in straight tunnel scenario in work [17] varies similarly, in which ASD becomes larger as the distance of $\mathrm{Tx}$ and $\mathrm{Rx}$ increases and others decrease at the same time. ASA is greater than others in the majority of the snapshots, which indicates that the impact of reflections from the side walls is significant on radio propagation. Multiantennas can be considered to implement a MIMO system so that the system capacity can be increased by achieving diversity and multiplexing. Besides, directional antennas can be deployed to drop the ASA and reduce the impact of Doppler spread on the channel.

Simulation results reveal that ASA, ESA, ASD, and ESD vary similarly in tunnels $b, c$, and $d$. The ASA is compared in tunnels $b, c$, and $d$ as shown in Figure 13. The maximum of ASA in tunnels $b, c$, and $d$ is $65.8^{\circ}, 123.9^{\circ}$, and $73.8^{\circ}$, respectively. It is obvious that ASA in tunnel $c$ is larger than that in tunnel $d$, whereas ASA in tunnel $b$ is the lowest at the majority of snapshots. This observation indicates that spatial fading is affected by the cross section. Furthermore, the side walls have a more significant impact on the channel in a narrow tunnel than a wider one. 


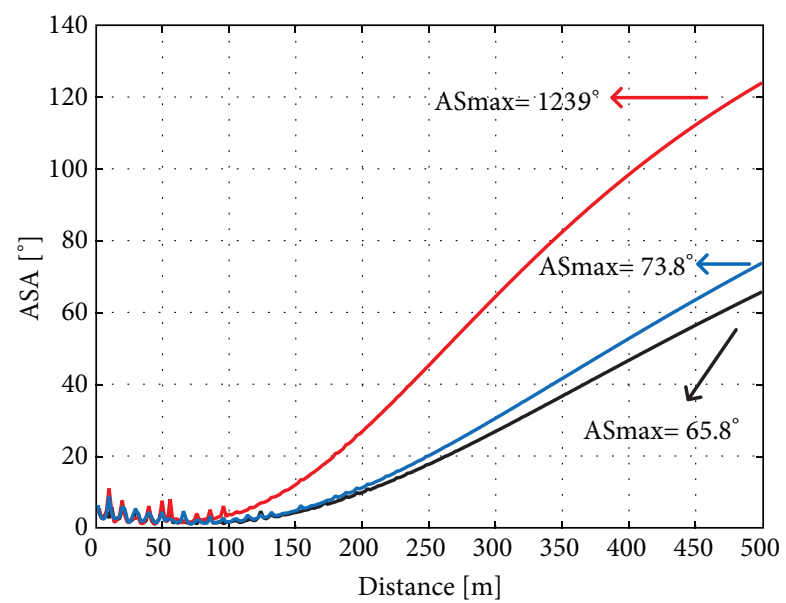

Tunnel b
Tunnel c
Tunnel d

Figure 13: ASA in tunnels $b, c$, and $d$.

\section{Conclusion}

In this paper, the radio channel characteristics of several tunnel scenarios were simulated with RT. The channel characteristics in different carrier frequencies and tunnel cross sections were analyzed. The key parameters, such as path loss, Rician K-factor, RMS delay spread, and angular spread were simulated and analyzed. The presence of the vehicle body introduces additional $35 \mathrm{~dB}$ of the path loss, which leads to the fluctuation and instability of the channel. $\mathrm{K}$-factor changes severely when the distance between Tx and $\mathrm{Rx}$ is smaller than $100 \mathrm{~m}$ and then decreases smoothly in far region. The channel has short delay spread (1.18 ns) due to strong LOS and the limited space. The side walls have a significant influence on radio propagation especially in a narrow tunnel. Therefore, the conclusion can be drawn that the channel experiences a severe and stable fading in long arched tunnels compared to other tunnel scenarios. The other parameters (i.e., Doppler spread, coherence time, antenna polarization, etc.) and leaky cable for coverage will be studied in the future work.

\section{Data Availability}

The data used to support the findings of this study are available from the corresponding author upon request.

\section{Conflicts of Interest}

The authors declare that they have no conflicts of interest.

\section{Acknowledgments}

This work was supported in part by the Fundamental Research Funds for the Central Universities (2018JBM079), the National Key Research and Development Program under
Grant 2016YFE0200900, the National Science and Technology Major Project (2015ZX03001027-003), the National Key R\&D Program of China under Grant no. 2016YFB1200100, the National Natural Science Foundation of China under Grant 61471030, the State Key Laboratory of Rail Traffic Control and Safety under Grant RCS2018ZZ006, and the Fundamental Research Funds for the Central Universities under Grant no. 2017YJS014.

\section{References}

[1] C.-X. Wang, A. Ghazal, B. Ai, Y. Liu, and P. Fan, "Channel measurements and models for high-speed train communication systems: a survey," IEEE Communications Surveys \& Tutorials, vol. 18, no. 2, pp. 974-987, 2015.

[2] D. W. Matolak, M. Berbineau, D. G. Michelson, and C. Chen, "Future railway communications [Guest Editorial]," IEEE Communications Magazine, vol. 53, no. 10, pp. 60-61, 2015.

[3] J. Marais, J. Beugin, and M. Berbineau, "A Survey of GNSSBased Research and Developments for the European Railway Signaling," IEEE Transactions on Intelligent Transportation Systems, vol. 18, no. 10, pp. 2602-2618, 2017.

[4] J. Zhang, Z. Zheng, Y. Zhang, J. Xi, X. Zhao, and G. Gui, "3D MIMO for 5G NR: Several Observations from 32 to Massive 256 Antennas Based on Channel Measurement," IEEE Communications Magazine, vol. 56, no. 3, pp. 62-70, 2018.

[5] T. S. Rappaport, Y. Xing, G. R. MacCartney, A. F. Molisch, E. Mellios, and J. Zhang, "Overview of millimeter wave communications for fifth-generation (5G) wireless networks-with a focus on propagation models," IEEE Transactions on Antennas and Propagation, no. 99, article 1, 2017.

[6] C. T. Neil, M. Shafi, P. J. Smith, P. A. Dmochowski, and J. Zhang, "Impact of Microwave and mmWave Channel Models on 5G Systems Performance," IEEE Transactions on Antennas and Propagation, vol. 65, no. 12, pp. 6505-6520, 2017.

[7] A. Hrovat, G. Kandus, and T. Javornik, "A survey of radio propagation modeling for tunnels," IEEE Communications Surveys \& Tutorials, vol. 16, no. 2, pp. 658-669, 2014.

[8] Y. Liu, A. Ghazal, C. Wang, X. Ge, Y. Yang, and Y. Zhang, "Channel measurements and models for high-speed train wireless communication systems in tunnel scenarios: a survey," Science China Information Sciences, vol. 60, no. 10, 2017.

[9] C. Zhou, "Ray tracing and modal methods for modeling radio propagation in tunnels with rough walls," IEEE Transactions on Antennas and Propagation, vol. 65, no. 5, pp. 2624-2634, 2017.

[10] K. Guan, B. Ai, Z. Zhong et al., "Measurements and Analysis of Large-Scale Fading Characteristics in Curved Subway Tunnels at $920 \mathrm{MHz}, 2400 \mathrm{MHz}$, and $5705 \mathrm{MHz}$," IEEE Transactions on Intelligent Transportation Systems, vol. 16, no. 5, pp. 2393-2405, 2015.

[11] K. Guan, Z. Zhong, B. Ai, and C. Briso-Rodriguez, "Statistic modeling for propagation in tunnels based on distributed antenna systems," in Proceedings of the 2013 IEEE International Symposium on Antennas and Propagation \& USNC/URSI National Radio Science Meeting, pp. 1920-1921, Orlando, FL, USA, July 2013.

[12] C. Briso-Rodriguez, J. M. Cruz, and J. I. Alonso, "Measurements and modeling of distributed antenna systems in railway tunnels," IEEE Transactions on Vehicular Technology, vol. 56, no. 5, pp. 2870-2879, 2007. 
[13] J. A. Castiblanco, D. Seetharamdoo, M. Berbineau et al., "Surface impedance boundary conditions in time domain for guided structures of arbitrary cross section with lossy dielectric walls," Institute of Electrical and Electronics Engineers. Transactions on Antennas and Propagation, vol. 63, no. 3, pp. 1086-1097, 2015.

[14] A. Hrovat, G. Kandus, and T. Javornik, "Four-slope channel model for path loss prediction in tunnels at $400 \mathrm{MHz}$," IET Microwaves, Antennas \& Propagation, vol. 4, no. 5, pp. 571-582, 2010.

[15] K. Guan, Z. D. Zhong, J. I. Alonso, and C. Briso-Rodríguez, "Measurement of distributed antenna systems at $2.4 \mathrm{GHz}$ in a realistic subway tunnel environment," IEEE Transactions on Vehicular Technology, vol. 61, no. 2, pp. 834-837, 2012.

[16] K. Guan, X. Lin, D. He et al., "Scenario modules and raytracing simulations of millimeter wave and terahertz channels for smart rail mobility," in Proceedings of the 2017 11th European Conference on Antennas and Propagation (EUCAP), pp. 113-117, Paris, France, March 2017.

[17] D. He, B. Ai, K. Guan et al., "Channel Measurement, Simulation, and Analysis for High-Speed Railway Communications in 5G Millimeter-Wave Band," IEEE Transactions on Intelligent Transportation Systems, no. 99, pp. 1-15, 2017.

[18] L. Zhang, C. Briso, J. R. O. Fernandez et al., "Delay spread and electromagnetic reverberation in subway tunnels and stations," IEEE Antennas and Wireless Propagation Letters, vol. 15, pp. 585$588,2016$.

[19] J. Zhang, C. Pan, F. Pei, G. Liu, and X. Cheng, "Threedimensional fading channel models: a survey of elevation angle research," IEEE Communications Magazine, vol. 52, no. 6, pp. 218-226, 2014.

[20] Nokia Networks, "Channel modelling for high speed leaky cable scenarios," in Proceedings of the 3GPP TSG-RAN WG4 Meeting \#76, R4-154106, Beijing, China, 2015.

[21] Huawei, "Channel models for the leaky cable," in Proceedings of the 3GPP TSG-RAN WG4 Meeting \#76, HiSilicon, R4-154241, Beijing, China, 2015.

[22] Huawei. HiSilicon, "Channel models for the leaky cable," in 3GPP TSG-RAN WG4 Meeting \#75, Japan, Fukuoka, 2015.

[23] Huawei, "TP: Channel model for leaky cable in tunnel," in Proceedings of the 3GPP TSG-RAN WG4 Meeting\#76, HiSilicon, R4-154242, Beijing, China, 2015.

[24] S. Priebe, towards THz communications: propagation studies, indoor channel modeling and interference investigations, Shaker Verlag, 2013.

[25] D. He, J. Yang, K. Guan et al., "Ray-tracing simulation and analysis of propagation for 3GPP high speed scenarios," in Proceedings of the 2017 11th European Conference on Antennas and Propagation (EUCAP), pp. 2890-2894, Paris, France, March 2017.

[26] Ningde high-speed railway tunnel covering research report, GCI Science Technology Co., Ltd, Guangzhou, China, Tech. Rep, 2008.

[27] N. Wang, "A uniform path-loss model by power balance theory (PBT) and its application on tunnels," in Proceedings of the 2010 9th International Symposium on Antennas Propagation and EM Theory, ISAPE 2010, pp. 481-484, China, December 2010.

[28] D. Didascalou, J. Maurer, and W. Wiesbeck, "Subway tunnel guided electromagnetic wave propagation at mobile communications frequencies," IEEE Transactions on Antennas and Propagation, vol. 49, no. 11, pp. 1590-1596, 2001.
[29] S. Shinozaki, M. Wada, A. Teranishi, H. Furukawa, and Y. Akaiwa, "Radio propagation characteristics in subway platform and tunnel in $2.5 \mathrm{GHz}$ band," in Proceedings of the 1995 6th IEEE International Symposium on Personal, Indoor and Mobile Radio Communications, PIMRC'95. Part 3 (of 3), pp. 1175-1179, September 1995.

[30] M. Choi, D. Kim, H. Jo, J. Yook, and H. Park, "Path-loss characteristics in subway tunnels at $2.65 \mathrm{GHz}$," Microwave and Optical Technology Letters, vol. 48, no. 2, pp. 383-386, 2006.

[31] Y. Zhang, Y. Liu, J. Sun, C. Wang, and X. Ge, "Impact of Different Parameters on Channel Characteristics in a High-Speed Train Ray Tracing Tunnel Channel Model," in Proceedings of the 2017 IEEE 85th Vehicular Technology Conference (VTC Spring), pp. 1-5, Sydney, NSW, June 2017.

[32] A. F. Molisch, Wireless Communications, IEEE-Wiley, 2nd edition, 2011.

[33] X. Cai, X. Yin, X. Cheng, and A. Perez Yuste, "An Empirical Random-Cluster Model for Subway Channels Based on Passive Measurements in UMTS," IEEE Transactions on Communications, vol. 64, no. 8, pp. 3563-3575, 2016.

[34] R. He, Z. Zhong, B. Ai et al., "Propagation channel measurements and analysis at $2.4 \mathrm{GHz}$ in subway tunnels," IET Microwaves, Antennas \& Propagation, vol. 7, no. 11, pp. 934-941, 2013. 


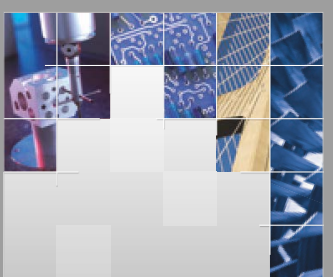

\section{Enfincering}
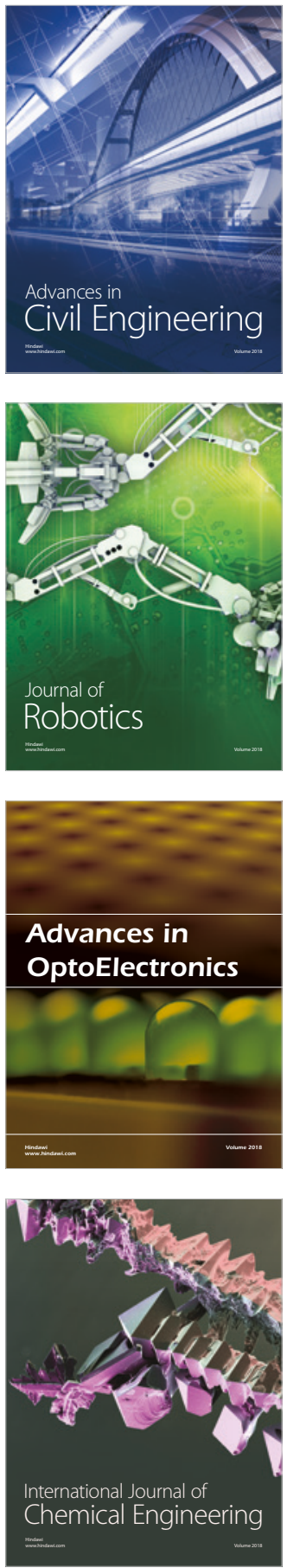

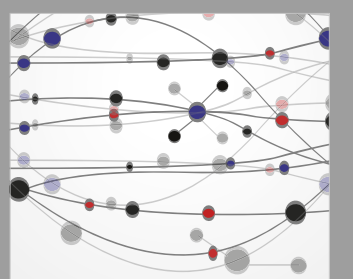

\section{Rotating \\ Machinery}

The Scientific World Journal

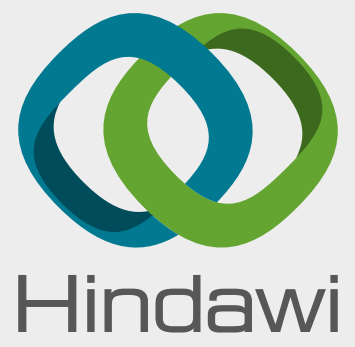

Submit your manuscripts at

www.hindawi.com
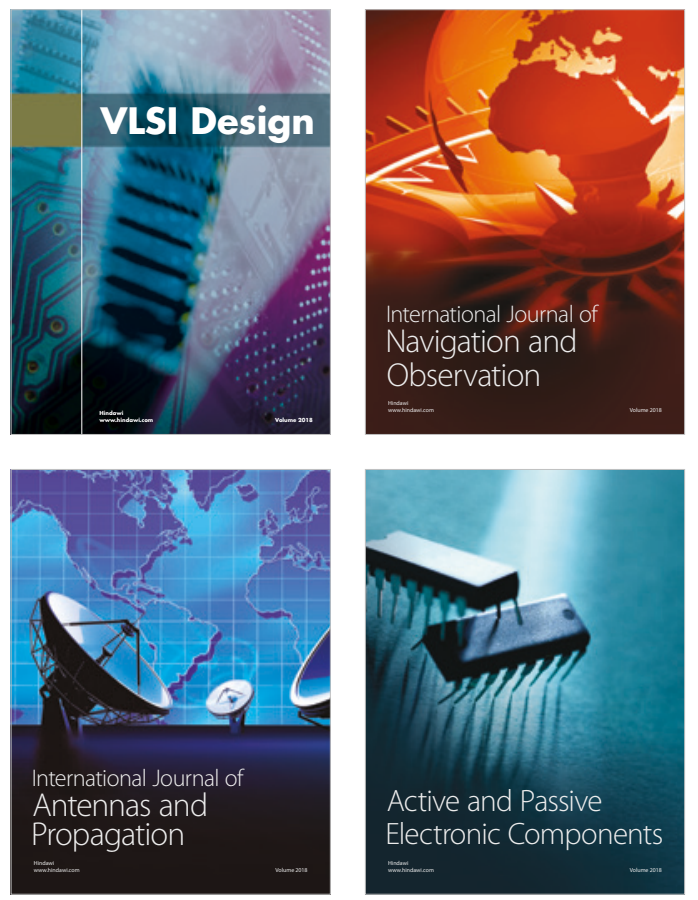
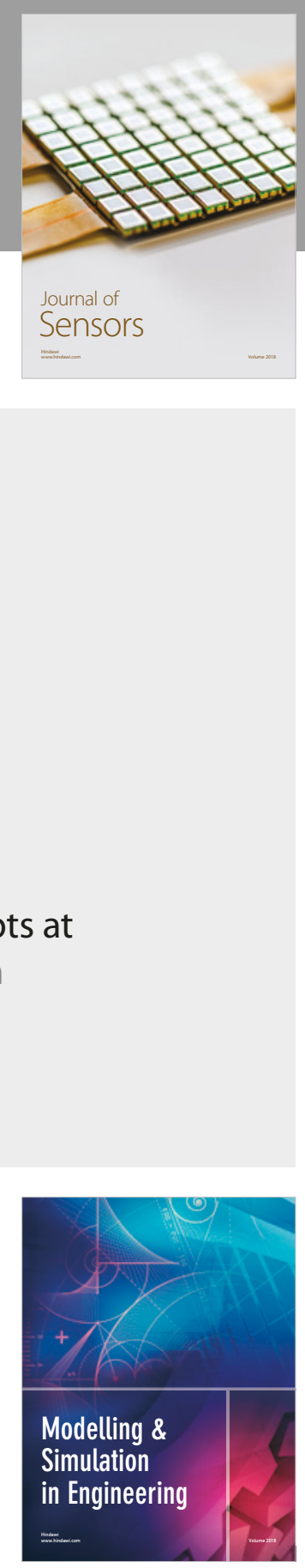

\section{Advances \\ Multimedia}
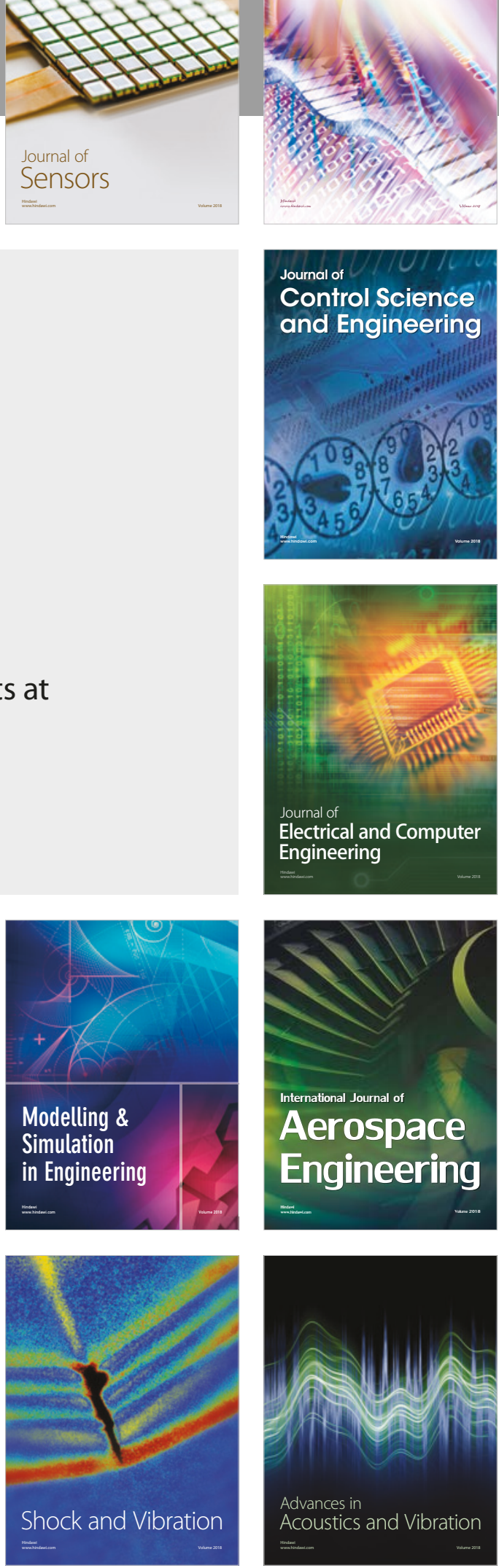